\title{
Diagnóstico e conduta no espessamento endometrial e pólipo endometrial em mulheres na pós-menopausa: Revisão Narrativa
}

\author{
Diagnostic and conduct in endometrial thickness and endometrial polyp in women in post- \\ menopause: Narrative Review
}

\author{
Diagnóstico y conducta del espesor endometrial y pólipo endometrial em post- \\ menopausia: Revisión narrativa
}

Aline Maria Freire Carvalho ${ }^{1 *}$, Graciete Helena Nascimento dos Santos ${ }^{1}$, Maria Tereza Freire Carvalho², Eduardo Moreira Dias ${ }^{1}$, Naylanny Gonçalves Torres Cunha².

\section{RESUMO}

Objetivo: Realizar uma revisão narrativa sobre método diagnóstico e conduta no espessamento e pólipo endometrial em mulheres na pós-menopausa. Métodos: trata-se de uma revisão de literatura narrativa conduzida nas bases de dados eletrônica: PubMed, utilizando o termo "Endometrial polyps" (pólipos endometriais), "Endometrial thickening" (espessamento endometrial) combinado com: "Post menopausal" (Pós-menopausa) e "management" (conduta/manejo). Para a seleção e avaliação dos artigos científicos foram estabelecidos critérios como: idiomas (português, inglês), período da publicação (2010 a 2019) e correlação com o tema do estudo proposto. Inicialmente foram encontradas 825 publicações, resultando em 19 artigos selecionados com base nos critérios de elegibilidade. O estudo não necessitou passar pelo Comitê de Ética em Pesquisa. Discussão: Estudos apontam uma tendência conservadora no manejo de pólipos endometriais assintomáticos em mulheres com baixo risco de malignização e indicação cirúrgica nas pacientes com fatores de risco, propondo 0 tratamento individualizado das pacientes. Conclusão: aumento da espessura endometrial em mulheres pós-menopáusicas com ou sem sangramento uterino anormal (AUB) é principalmente devido a lesões benignas, como pólipos e miomas submucosos. Histeroscopia é um método seguro e confiável para avaliar e tratar essas lesões. Há uma tendência atual de indicação de gestão individualizada para essas pacientes, devendo considerar as características de cada caso.

Palavras chave: Espessamento endometrial, Pólipo endometrial, Pós-menopausa.

\section{ABSTRACT}

Objective: Carry out a Narrative Review on the diagnostic method and thickening and endometrial polyp in postmenopausal women. Methods: $t$ is a review of narrative literature conducted in the electronic databases Pubmed; using the term "endometrial polyps", "endometrial thickening" combined with "post-menopausal" and "management". For the selection and evaluation of scientific articles, criteria such as: languages (Portuguese, English), publication period (2010 to 2019) and correlation with the theme of the proposed study were established. Initially, 825 publications were found, resulting in 19 articles selected based on the eligibility criteria. The study did not need to go through the Research Ethics Committee. Discussion: Studies indicate a conservative trend in the management of asymptomatic endometrial polyps in women with low risk of malignancy and surgical indication in patients with risk factors, proposing the individualized treatment of patients. Conclusion: Increased endometrial thickness in postmenopausal women with or without abnormal uterine bleeding (AUB) is mainly due to benign lesions such as polyps and submucous myomas. Hysteroscopy

1Universidade Federal do Maranhão (UFMA), São Luís-Maranhão. *E-mail: alinefreire.c@gmail.com

2Universidade Estadual do Maranhão (UEMA), Bacabal - MA. 
is a safe and reliable method to evaluate and treat these lesions. There is a current trend of indication of individualized management for these patients, with the characteristics of each case.

Key words: Endometrial thickening, Endometrial polyp, Post-menopause.

\section{RESUMEN}

Objetivo: realizar una revisión narrativa sobre el método de diagnóstico y el tratamiento del engrosamiento y pólipo endometrial en mujeres posmenopáusicas. Métodos: Esta es una revisión de la literatura narrativa realizada en bases de datos electrónica: Pubmed, utilizando el término "pólipos endometriales", "engrosamiento endometrial" (engrosamiento endometrial) combinado con: "Postmenopáusica" y "manejo". Para la selección y evaluación de artículos científicos se establecieron criterios como: idiomas (portugués, inglés), período de publicación (2010 a 2019) y correlación con el tema del estudio propuesto. Inicialmente se encontraron 825 publicaciones, lo que resultó en 19 artículos seleccionados en función de los criterios de elegibilidad. El estudio no tuvo que pasar por el Comité de Ética de Investigación. Discusión: Los estudios apuntan a una tendencia conservadora en el manejo de pólipos endometriales asintomáticos en mujeres con bajo riesgo de malignidad e indicación quirúrgica en pacientes con factores de riesgo, proponiendo un tratamiento individualizado de pacientes. Conclusión: El aumento del grosor endometrial en mujeres posmenopáusicas con o sin sangrado uterino anormal (AUB) se debe principalmente a lesiones benignas como pólipos y fibromas submucosos. La histeroscopia es un método seguro y confiable para evaluar y tratar estas lesiones. Existe una tendencia actual de indicación de manejo individualizado para estos pacientes, con las características de cada caso.

Palabras clave: Engrosamiento endometrial, Pólipo endometrial, Posmenopausia.

\section{INTRODUÇÃO}

Os pólipos endometriais são anormalidades estruturais e epiteliais comuns do endométrio frequentemente encontradas por ginecologistas nas práticas de consultórios. Clark TJ e Stevenson H (2017) relatam que esses crescimentos endometriais podem ser encontrados incidentalmente em até $10 \%$ a $15 \%$ das mulheres assintomáticas e entre $20 \%$ a $30 \%$ das mulheres com sangramento uterino anormal (AUB). Dada a prevalência em pacientes com $A U B$, os pólipos são considerados parte da classificação da Federação Internacional de Ginecologia e Obstetrícia (FIGO) para causas de sangramento uterino anormal (AUB) PALM-COEIN (MUNRO MG, et al., 2018). Eles podem causar uma variedade de padrões de sangramento anormais, provavelmente devido a pequenas artérias musculares encontradas nos crescimentos, mas também podem ser completamente assintomáticos (VINAY K, et al., 2010). As patologias uterinas estruturais geralmente causam menstruação pesada ou prolongada, embora mensalmente (FRITZ MA, et al., 2011) mas, os pólipos também podem causar sangramento intermenstrual ou pós-menopáusico (CLARK TJ e STEVENSON H, 2017). Clark TJ e Stevenson H (2017) acrescentam em suas falas que os pólipos endometriais são geralmente benignos, mas em 0,5\% a 3\% dos casos são malignos. Vroom AJ et al. (2019) chamam atenção em seus estudos para as mulheres na pós-menopausa que têm sangramento uterino anormal (AUB), o risco de encontrar patologia pré-cancerosa nos pólipos pode chegar a $6 \%$.

Poggio GA et al. (2018) relatam que mulheres com suspeita de pólipo endometrial são tipicamente avaliadas com estudo de imagem pélvica (ultrassonografia) ou histeroscopia onde, a ultrassonografia transvaginal (TVUS) é o exame de imagem selecionado de primeira escolha para avaliar mulheres com sangramento uterino anormal. Já as mulheres pós-menopáusicas ou que são candidatas a um manejo expectante é sugerida histeroscopia diagnóstica (GONÇALVES KFS, 2016).

Wanderley MD et al. (2016), afirmam que em mulheres na menopausa (até 12 meses com ausência da menstruação) o endométrio apresenta-se com uma fina linha ecogênica linear e homogênea, medindo até 5 $\mathrm{mm}$. Quando em uso de terapia hormonal, o eco endometrial pode atingir até $10 \mathrm{~mm}$, com padrão homogêneo e ecogênico. Algumas literaturas referem que apesar do ponto de corte do espessamento endometrial ser em 
torno de 4 a $5 \mathrm{~mm}$, deve ser ressaltado que quando há essa alteração na ecografia, não necessariamente significa que se tem uma patologia do endométrio confirmada, mas sim um estudo evidenciando a necessidade de complementação diagnóstica, como por exemplo, através da histeroscopia (SADRO CT, 2016). Naquelas mulheres na pós-menopausa (após os 12 meses com ausência da menstruação) as causas mais comuns de sangramento anormal são o endométrio atrófico, hiperplasia endometrial, câncer endometrial, pólipos e fibromas submucosos (SADRO CT, 2016).

O ultrassom é indicado como um método de imagem de diagnóstico seguro, não invasivo, portátil, barato e facilmente reprodutível que fornece excelentes resultados quando realizado por um operador treinado (POGGIO GA, et al., 2017) Outro método diagnóstico utilizado para investigação relatada pelos autores é a histeroscopia cirúrgica e ambulatorial (SILVEIRA DF, et al., 2017) Atualmente, o método propedêutico disponível mais apropriado para a avaliação da cavidade uterina em mulheres com suposto espessamento endometrial, sejam elas assintomáticas ou com sangramento vaginal, é a histeroscopia (POGGIO GA, et al., 2017)

Desta forma, o objetivo desse estudo foi apresentar uma revisão narrativa de literatura acerca dos diagnósticos e conduta mais adequada referente ao espessamento endometrial e pólipos endometriais em mulheres pós-menopáusicas.

\section{MÉTODOS}

Após análise e cruzamento dos descritores e duplicidade de artigos foram obtidos 1.079 artigos e, destes analisando o objeto de estudo, 121 artigos foram incluídos. Assim, em conformidade com os critérios de inclusão da pesquisa: ano da publicação; objeto e objetivo da pesquisa; disponibilidade (texto integral); idiomas: português e inglês, resultaram 18 artigos (Figura 1 e Tabela 1).

Figura 1 - Estratégia de busca nas bases de dados.

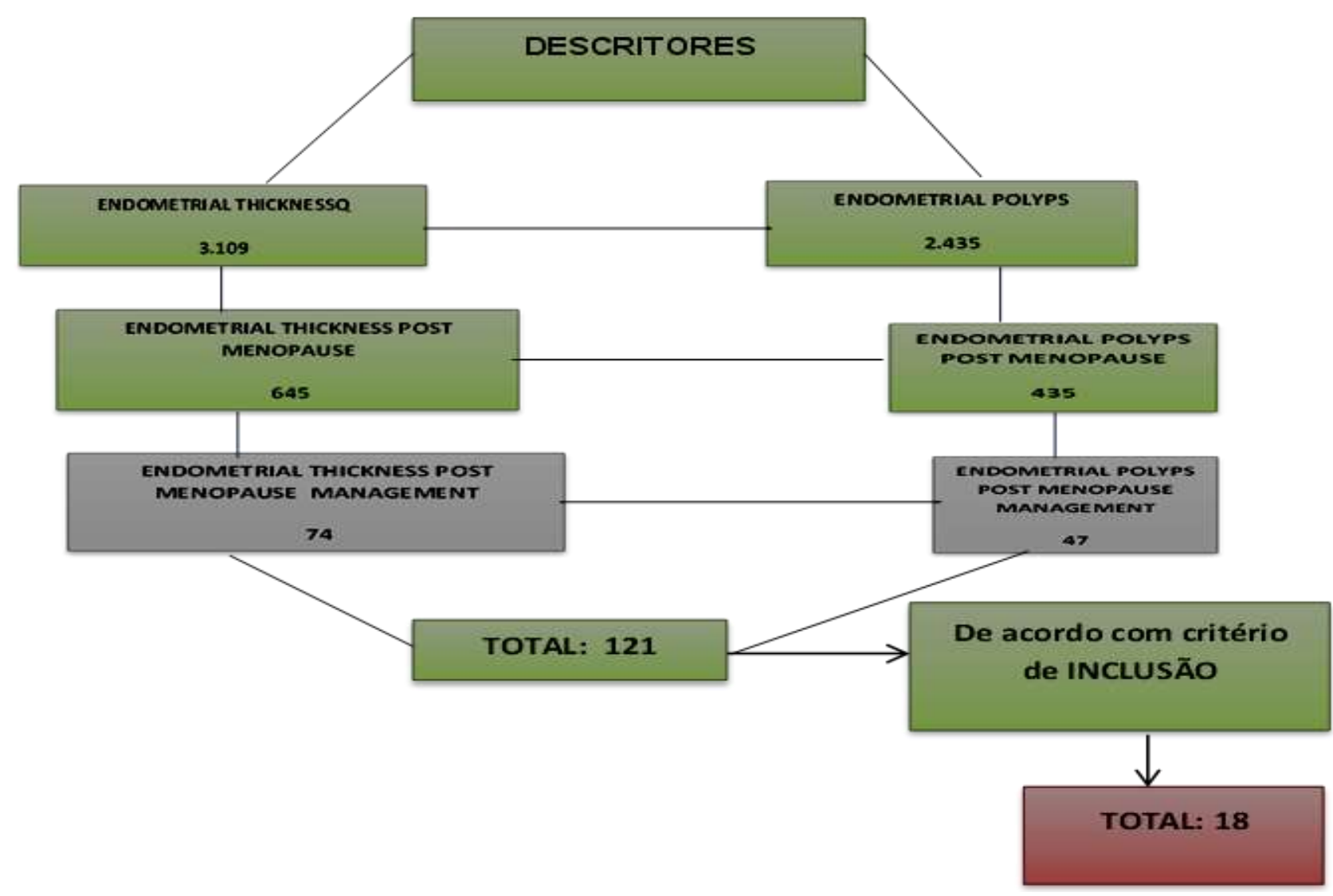

Fonte: Carvalho AMF et al. (2019) 
Realizou-se uma revisão narrativa da literatura que foi construída a partir de uma das principais bases de dados eletrônicos: PUBMED via MEDLINE empregando os descritores (DESC) / palavras-chaves: endometrial polyps / pólipo endometrial AND thickening endometrial / espessamento endometrial AND postmenopausa / pós menopausa AND manegement / conduta/manejo.

O levantamento dos trabalhos foi de acordo com os critérios de inclusão: idioma inglês (descritores-DESC) e português (palavras-chaves); disponibilidade (texto integral); objeto e objetivo do estudo; tempo/ano (2014 a 2019). Assim, realizada a triagem das obras, o material selecionado foi lido e analisado possibilitando a elaboração da revisão teórica construída. Inicialmente, a partir da busca de dados no PUBMED foram obtidos 5.544 artigos conforme a utilização dos descritores/palavras-chaves de busca da pesquisa.

\section{RESULTADO E DISCUSSÃO}

Quanto aos pólipos endometriais, podem ser encontrados incidentalmente em até $10 \%$ a $15 \%$ das mulheres assintomáticas e entre $20 \%$ a $30 \%$ das mulheres com sangramento uterino anormal (AUB) (IMUDIA NA e NEW EP, 2019). Os pólipos são considerados como lesões proliferativas focais com formação circunscrita que fazem protrusão para a cavidade do endométrio. Podem ser tanto sésseis como pediculados, ovoides ou alongados, com dimensões de 2 a 4 mm e até passar pelo óstio cervical (SILVEIRA DF, et al., 2017).

Segundo a literatura, a taxa de maliguinização de pólipos endometriais está entre $0 \%$ e 4,8\%, portanto, de baixo potencial de malignidade (CARVALHO GA, 2019; FARIA F e FERNANDES ES, 2017; GONÇALVES KFS, 2016; BILLINGSLEY CC, 2015). Clinicamente, os pólipos endometriais podem ou não apresentar sintomas. Mulheres com sintomas geralmente apresentam queixa de sangramento uterino anormal - sendo esse um importante fator de risco para maior incidência de câncer de endométrio juntamente com a pósmenopausa (CARVALHO GA, 2019; GHOUBARA A, et al., 2017).

Observou-se que os pólipos endometriais são frequentemente os culpados da AUB, nesse caso, 0 tratamento é necessário para diminuir os sintomas da paciente e confirmar o diagnóstico, especialmente porque os pólipos benignos e malignos somente podem ser distinguidos de forma confiável com exames de imagem; sendo a histeroscopia com polipectomia o tratamento de escolha, servindo tanto como procedimento diagnóstico como terapêutico (GHOUBARA A, et al., 2018; IMUDIA NA e NEW EP, 2019).

Quanto ao espessamento endometrial na pós-menopausa, em uma paciente hígida, o endométrio é representado por uma linha delgada ao eco. Quando sua medida é inferior a $3 \mathrm{~mm}$ não há necessidade de estudo patológico. Se espesso e ultrapassando a medida de $5 \mathrm{~mm}$ é denominado como endométrio ativo e deve ser investigado por biópsia ou histeroscopia ambulatorial (SILVEIRA DF, et al., 2017; FARIA F e FERNANDES ES, 2017; GHOUBARA A, et al., 2018). Deve-se levar em consideração o risco naquelas pacientes em que se encontram na pós-menopausa, com sangramento vaginal e que ao ultrassom 0 endométrio apresenta-se com espessamento de mais de $5 \mathrm{~mm}$, indicando a necessidade de biópsia. Já nas pacientes que se encontram na pós-menopausa, porém sem sangramento, é indicado a solicitação da biópsia caso o endométrio apresente uma espessura de $9 \mathrm{~mm}$ ou superior a este valor (SADRO CT, 2016)

Quanto aos exames de imagens, o ultrassom foi descrito como um método de imagem de diagnóstico seguro, não invasivo, portátil, barato e facilmente reprodutível que fornece excelentes resultados quando realizado por um operador treinado (POGGIO GA, et al., 2017).

O ultrassom transvaginal é o método de escolha para investigação inicial das doenças endometriais; tanto nas pacientes com sangramento genital, quanto nas mulheres assintomáticas principalmente na pósmenopausa (SILVEIRA DF, et al., 2017; CARVALHO GA, 2019).

A histeroscopia combinada com a biópsia direta é o método mais utilizado devido a sua alta sensibilidade e especificidade, além de permitir biópsias dirigidas, com menor risco de erro de amostragem (IMUDIA NA e NEW EP, 2019; SADRO CT, 2016). A histeroscopia é considerada padrão-ouro para o diagnóstico e tratamento do pólipo endometrial (IMUDIA NA e NEW EP, 2019; MEENA J, et al., 2017; WANDERLEY MD, et al., 2016). 
Tabela 1 - Técnica resumida de alguns estudos selecionados de acordo com os critérios de inclusão, na data base do PubMed observando as variáveis: tempo (ano), autor/autores, título, objetivo e conclusão do trabalho.

\begin{tabular}{|c|c|c|c|}
\hline Trabalhos (Autor) & Título & Objetivo & Conclusão \\
\hline Carvalho GA (2019) & $\begin{array}{l}\text { Câncer de endométrio: quais } \\
\text { os fatores associados com } \\
\text { recidiva }\end{array}$ & $\begin{array}{l}\text { Caracterizar variáveis clínicas e } \\
\text { histopatológicas das pacientes que } \\
\text { apresentaram recidiva de câncer de } \\
\text { endométrio após o tratamento cirúrgico } \\
\text { primário. }\end{array}$ & $\begin{array}{l}\text { Concluíram que a biopsia de endométrio é o } \\
\text { método padrão-ouro para diagnostico de } \\
\text { neoplasias. }\end{array}$ \\
\hline Jiang $T$, et al (2019) & $\begin{array}{l}\text { As lesões endometriais } \\
\text { requerem remoção? Um } \\
\text { estudo retrospectivo. }\end{array}$ & $\begin{array}{l}\text { Avaliar o manejo de lesões intrauterinas } \\
\text { assintomáticas detectadas pela } \\
\text { ultrassonografia. }\end{array}$ & $\begin{array}{l}\text { O TVOS foi altamente sensível (94\%) para pólipos } \\
\text { pré-menopáusicos e } 92,7 \% \text { para pólipos pós } \\
\text { menopausa. }\end{array}$ \\
\hline Imudia AN, New EP (2019) & $\begin{array}{l}\text { Pólipos endometriais: } \\
\text { manejo em consultório }\end{array}$ & $\begin{array}{l}\text { Avaliar a eficácia da remoção } \\
\text { histeroscópica de pólipos endometriais }\end{array}$ & $\begin{array}{l}\text { Pólipos endometriais encontrados na } \\
\text { ultrassonografia durante uma investigação para } \\
\text { AUB são comumente encontrados e facilmente } \\
\text { gerenciados com procedimentos histeroscópicos } \\
\text { baseados em consultório. Dado o risco subjacente } \\
\text { de malignidade nos pólipos endometriais, que } \\
\text { pode ser tão baixo quanto } 0,5 \% \text { a } 3 \% \text { em todas as } \\
\text { mulheres diagnosticadas com pólipos } \\
\text { (GHOUBARA A et al., 2018) ou até } 6 \% \text { em } \\
\text { mulheres pós-menopáusicas com sangramento } \\
\text { (VROOM AJ et al, 2019) a remoção é fortemente } \\
\text { recomendada com polipectomia histeroscópica } \\
\text { para eventual histopatológico diagnóstico. }\end{array}$ \\
\hline Vroom J, et al. (2019) & $\begin{array}{l}\text { Acurácia diagnóstica dá } \\
\text { sono-histerografia com } \\
\text { contraste salino na detecção } \\
\text { de pólipos endometriais em } \\
\text { mulheres com sangramento } \\
\text { na pós-menopausa: revisão } \\
\text { sistemática e metanálise. }\end{array}$ & $\begin{array}{l}\text { Avaliar a precisão da sono-histerografia } \\
\text { com contraste salino (SCSH) para o } \\
\text { diagnóstico de pólipos endometriais em } \\
\text { mulheres com sangramento na pós- } \\
\text { menopausa. }\end{array}$ & $\begin{array}{l}\text { Desde que o exame SCSH seja de ótima } \\
\text { qualidade, pode ser considerado como um método } \\
\text { para estratificar as mulheres com o PMB para } \\
\text { posterior investigação e tratamento de diagnóstico } \\
\text { com histeroscopia. Em mulheres sem suspeita de } \\
\text { uma lesão polipóide em SCSH e com endométric } \\
\text { benigno amostra, a conduta expectante deve se } \\
\text { considerada. }\end{array}$ \\
\hline
\end{tabular}




\begin{tabular}{|c|c|c|c|}
\hline Ghoubara A, et al. (2018) & $\begin{array}{l}\text { Espessamento endometrial } \\
\text { em mulheres pós- } \\
\text { menopáusicas } \\
\text { assintomáticas } \\
\text { determinando um limiar ideal } \\
\text { para previsão de hiperplasia } \\
\text { atípica e câncer. }\end{array}$ & $\begin{array}{l}\text { Realizar uma revisão de literatura para } \\
\text { abordar o dilema do manejo de mulheres } \\
\text { pós-menopausadas assintomáticas com } \\
\text { um achado incidental de endométrio } \\
\text { espessado }\end{array}$ & $\begin{array}{l}\text { Este estudo mostrou que a prevalência da } \\
\text { patologia é alta o suficiente para justificar a } \\
\text { investigação e para o problema clínico não ser } \\
\text { banalizado pelas mulheres e pelos médicos. }\end{array}$ \\
\hline Ghoubara A, et al. (2017) & $\begin{array}{l}\text { Preditores de malignidade } \\
\text { em pólipos endometriais: } \\
\text { estudo de } 421 \text { mulheres } \\
\text { com sangramento pós- } \\
\text { menopausa. }\end{array}$ & $\begin{array}{l}\text { Quantificar a prevalência e identificar os } \\
\text { fatores preditores de hiperplasia e câncer } \\
\text { em pólipos. }\end{array}$ & $\begin{array}{l}\text { A prevalência de hiperplasia e câncer em pólipos } \\
\text { benignos é alta. Os preditores independentes são } \\
\text { o índice de massa corporal e a espessura } \\
\text { endometrial. A remoção de pólipos pode ser } \\
\text { justificada até que critérios morfológicos } \\
\text { histeroscópicos que possam prever com } \\
\text { segurança o resultado sejam estabelecidos em } \\
\text { pesquisas futuras. }\end{array}$ \\
\hline Meena J, et al. (2017) & $\begin{array}{l}\text { Relato de caso de pólipo } \\
\text { endometrial gigante em } \\
\text { mulher pós-menopáusica } \\
\text { assintomática }\end{array}$ & $\begin{array}{l}\text { Relatar um caso de pólipo endometrial } \\
\text { gigante em mulher na pós-menopausa } \\
\text { sem sangramento vaginal e uso de } \\
\text { hormônios ou drogas. }\end{array}$ & $\begin{array}{l}\text { A mulher na pós-menopausa nem sempre } \\
\text { apresentará sintomas e a USG também pode errar } \\
\text { o diagnóstico com frequência, por isso é } \\
\text { necessária uma avaliação adequada com a } \\
\text { histeroscopia, que é o padrão-ouro para o } \\
\text { diagnóstico e tratamento do pólipo endometrial. }\end{array}$ \\
\hline Faria F e Fernandes ES (2017) & $\begin{array}{lr}\text { Perfil das r pacientes } \\
\text { investigadas por } \\
\text { sangramento r pós- } \\
\text { menopausa no Hospital Júlia } \\
\text { Kubitschek nos anos de } \\
2010 \text { a 2014. }\end{array}$ & $\begin{array}{l}\text { Identificar em que faixa de espessamento } \\
\text { endometrial há mais casos de câncer de } \\
\text { endométrio e traçar um perfil das } \\
\text { pacientes submetidas aos procedimentos } \\
\text { de amostragem de endométrio. }\end{array}$ & $\begin{array}{l}\text { A média de espessura endometrial encontrada em } \\
\text { casos de câncer de endométrio foi de } 12,4 \mathrm{~mm} \text {, } \\
\text { variando entre } 5,3 \mathrm{~mm} \text { e } 19,5 \mathrm{~mm} \text { e não mostrou } \\
\text { associação entre esses fatores. }\end{array}$ \\
\hline Silveira DF, et al. (2017) & $\begin{array}{lr}\text { Avaliação } & \text { ultrassonográfica } \\
\text { no } & \text { espessamento } \\
\text { endometrial: } & \text { revisão de } \\
\text { literatura } & \end{array}$ & $\begin{array}{l}\text { Avaliar o real papel da ecografia pélvica } \\
\text { na visualização das doenças } \\
\text { endometriais. }\end{array}$ & $\begin{array}{l}\text { A ecografia é o exame primordial e elementar na } \\
\text { investigação de pacientes com sangramento } \\
\text { uterino anormal ou com suspeita de outras } \\
\text { doenças que alteram o padrão endometrial, } \\
\text { conduzindo o prognóstico do quadro investigado. }\end{array}$ \\
\hline
\end{tabular}




\begin{tabular}{|c|c|c|c|}
\hline Poggio GA, et al. (2017) & $\begin{array}{l}\text { Primeiro, o ultrassom: por } \\
\text { que, como e quando? }\end{array}$ & $\begin{array}{l}\text { Sensibilizar os médicos sobre o uso da } \\
\text { ultrassonografia como primeira } \\
\text { ferramenta de diagnóstico. }\end{array}$ & $\begin{array}{l}\text { Os autores reafirmam o valor do ultrassom como } \\
\text { primeiro método de escolha para diagnóstico, } \\
\text { porém, devem estar atreladas as habilidades e } \\
\text { conhecimento do profissional na radiologia. }\end{array}$ \\
\hline Van Hanegem N, et al. (2017) & $\begin{array}{l}\text { Análise diagnóstica para } \\
\text { sangramento pós- } \\
\text { menopausa: um estudo } \\
\text { controlado randomizado. }\end{array}$ & $\begin{array}{l}\text { Avaliar a eficácia da histeroscopia para a } \\
\text { detecção e tratamento de pólipos } \\
\text { endometriais versus o tratamento } \\
\text { expectante em mulheres com } \\
\text { sangramento na pós - menopausa } \\
\text { (PMB), um endométrio espessado e } \\
\text { amostragem endometrial benigna. }\end{array}$ & $\begin{array}{l}\text { Em mulheres com PMB, a histeroscopia não reduz } \\
\text { o sangramento recorrente, mas é necessária para } \\
\text { detectar malignidade focal. }\end{array}$ \\
\hline Bueloni-Dias FN, et al. (2016) & $\begin{array}{l}\text { Síndrome metabólica como } \\
\text { preditor de pólipos } \\
\text { endometriais em mulheres } \\
\text { na pós-menopausa. }\end{array}$ & 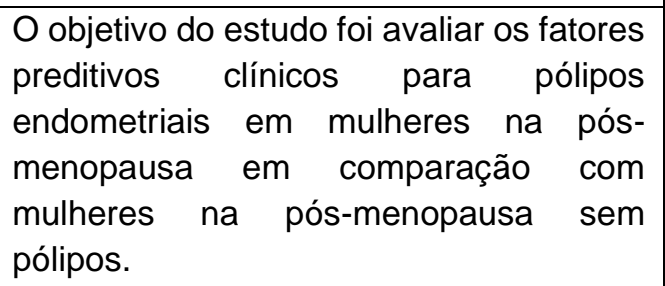 & $\begin{array}{l}\text { Em mulheres na pós-menopausa, obesidade, } \\
\text { dislipidemia, hiperglicemia e presença de SM } \\
\text { foram fatores preditivos para pólipos endometriais. }\end{array}$ \\
\hline Spadoto-Dias D, et al. (2016) & $\begin{array}{lrr}\text { O valor da } & \text { biópsia } \\
\text { histeroscópica } & \text { no } \\
\text { diagnóstico de } & \text { pólipos } \\
\text { endometriais. } & & \\
\end{array}$ & $\begin{array}{l}\text { Estabelecer uma correlação entre os } \\
\text { resultados histológicos de biopsias } \\
\text { endometriais em consultórios, } \\
\text { histeroscopicamente guiadas e cegas, } \\
\text { com os espécimes de polipectomia } \\
\text { cirúrgica. }\end{array}$ & $\begin{array}{l}\text { Este estudo preliminar demonstrou que as biópsias } \\
\text { realizadas em consultórios tiveram baixa acurácia } \\
\text { diagnóstica para pólipos endometriais quando } \\
\text { comparados com os espécimes de polipectomia. }\end{array}$ \\
\hline Gonçalves KFS (2016) & $\begin{array}{l}\text { Conduta } \quad \text { em pólipos } \\
\text { endometriais assintomáticos: } \\
\text { revisão sistemática de } \\
\text { literatura. }\end{array}$ & $\begin{array}{l}\text { Realizar uma revisão sistemática de } \\
\text { literatura para avaliar a conduta mais } \\
\text { adequada em pólipos endometriais } \\
\text { assintomáticos. }\end{array}$ & $\begin{array}{l}\text { Concluíram que o aumento da espessura } \\
\text { endometrial em mulheres pós-menopáusicas com } \\
\text { ou sem AUB é principalmente devido a lesões } \\
\text { benignas, como pólipos e miomas submucosos. A } \\
\text { histeroscopia é um método seguro e confiável para } \\
\text { avaliar e tratar essas lesões. }\end{array}$ \\
\hline
\end{tabular}




\begin{tabular}{|c|c|c|c|}
\hline Sarvi F, et al. (2016) & $\begin{array}{l}\text { Histeroscopia: um método } \\
\text { necessário para detectar } \\
\text { patologias uterinas em } \\
\text { mulheres pós-menopausa } \\
\text { com sangramento uterino } \\
\text { anormal ou aumento da } \\
\text { espessura endometrial. }\end{array}$ & $\begin{array}{l}\text { Investigar os achados histológicos e } \\
\text { histeroscópicos de mulheres pós- } \\
\text { menopáusicas com sangramento uterino } \\
\text { e mulheres assintomáticas com aumento } \\
\text { da espessura endometrial igual ou } \\
\text { superior a } 5 \mathrm{~mm} \text {. }\end{array}$ & $\begin{array}{l}\text { Concluíram que aumento da espessura } \\
\text { endometrial em mulheres pós-menopáusicas com } \\
\text { ou sem AUB é principalmente devido a lesões } \\
\text { benignas, como pólipos e miomas submucosos. A } \\
\text { histeroscopia o método confiável e segura para } \\
\text { avaliar e tratar essas lesões. }\end{array}$ \\
\hline Sadro CT (2016) & $\begin{array}{l}\text { Imagem do endométrio: um } \\
\text { ensaio pictórico }\end{array}$ & $\begin{array}{l}\text { Rever a aparência normal e anormal do } \\
\text { endométrio e diagnóstico para descrever } \\
\text { e avaliar sangramento vaginal anormal e } \\
\text { espessura endometrial anormal }\end{array}$ & $\begin{array}{l}\text { A modalidade de imagem mais comumente } \\
\text { utilizada para descrever e avaliar endométrio é a } \\
\text { ultrassonografia pélvica com técnicas } \\
\text { transabdominal e transvaginal. Métodos de } \\
\text { imagens adicionais incluem: histerossonografia e a } \\
\text { ressonância magnética. }\end{array}$ \\
\hline Billingsley CC, et al. (2015) & $\begin{array}{l}\text { O uso da ultrassonografia } \\
\text { transvaginal no câncer de } \\
\text { endométrio tipo II. }\end{array}$ & $\begin{array}{l}\text { Determinar o uso da ultrassonografia } \\
\text { transvaginal (TVUS) em mulheres na } \\
\text { pós-menopausa com câncer endometrial } \\
\text { tipo II }\end{array}$ & $\begin{array}{l}\text { O uso de TVUS, que tem valor no câncer tipo I, é } \\
\text { limitado no câncer de endométrio tipo II. Portanto, } \\
\text { a amostragem endometrial deve ser incluída na } \\
\text { avaliação de todas as mulheres com sangramento } \\
\text { na pós-menopausa, independentemente da } \\
\text { espessura do EMS. }\end{array}$ \\
\hline Bueloni-Dias FN, et al. (2014) & $\begin{array}{l}\text { Fatores preditivos para a } \\
\text { ocorrência de pólipos } \\
\text { endometriais em mulheres } \\
\text { na pós-menopausa. }\end{array}$ & $\begin{array}{l}\text { Avaliar os fatores clínicos preditivos para } \\
\text { o desenvolvimento de pólipos } \\
\text { endometriais em mulheres na pós- } \\
\text { menopausa. }\end{array}$ & $\begin{array}{l}\text { Em mulheres na pós-menopausa, obesidade, } \\
\text { dislipidemia, hiperglicemia e presença de } \\
\text { síndrome metabólica foram fatores preditivos para } \\
\text { o desenvolvimento de pólipos endometriais. }\end{array}$ \\
\hline Hebbar S, et al. (2014) & $\begin{array}{l}\text { Fatores que influenciam a } \\
\text { espessura endometrial na } \\
\text { pós-menopausa. }\end{array}$ & $\begin{array}{l}\text { Estudar os vários fatores que influenciam } \\
\text { a espessura endometrial em mulheres na } \\
\text { pós-menopausa }\end{array}$ & $\begin{array}{l}\text { Este estudo sugere que a paridade, IMC, presença } \\
\text { de mioma, uso de tamoxifeno, volume uterino, } \\
\text { volume ovariano e estradiol sérico influenciam o } \\
\text { TE em mulheres na pós-menopausa. }\end{array}$ \\
\hline
\end{tabular}


Quanto à conduta mais adequada em pólipos endometriais e espessamentos endometriais, os estudos apontam para uma tendência conservadora no manejo de pólipos endometriais assintomáticos em mulheres com baixo risco de malignização e indicação cirúrgica nas pacientes com fatores de risco, propondo o tratamento individualizado das pacientes. Alguns estudos defendem a ideia de que o USG transvaginal deve ser o método de escolha na investigação inicial. Nesse mesmo cenário, a histeroscopia é método padrão ouro tanto para o diagnóstico como para o tratamento de pólipos endometriais, todavia, não existe consenso absoluto sobre o manejo de pólipos (ARAÚJO FM, et al., 2019; SARVI F, et al., 2016; GONÇALVES KFS, 2016).

Existem referências do uso da solução salina contraste sonohysterography (SCSH) para o diagnóstico de pólipos endometriais em mulheres com sangramento pós-menopausa, onde, desde que o exame SCSH seja de ótima qualidade, pode ser considerado como um método para estratificar mulheres com sangramento pósmenopausa para posterior investigação diagnóstica e tratamento com histeroscopia (VROOM AJ, et al., 2019). Alguns estudos relatam que as biópsias endometriais em consultório tinham baixa precisão diagnóstica para pólipos endometriais em comparação com espécimes de polipectomia cirúrgica (SPADOTO-DIAS D, et al., 2016).

Outros autores preconizam como modalidade de imagem mais utilizada para avaliar o endométrio a ultrassonografia pélvica com técnicas transabdominal e transvaginal e os métodos de imagem adicionais incluindo histerossonografia e ressonância magnética; já outros estudos relatam que idealmente, após investigação inicial com ultrassonografia transvaginal, a biópsia guiada da lesão deve ser realizada por meio de histeroscopia, sempre que necessário, a fim de melhorar a acurácia diagnóstica e o posterior manejo clínico (WANDERLEY MD, et al., 2016).

Um estudo recente avaliando a eficácia da remoção histeroscópica de pólipos endometriais demonstrou que a histeroscopia de consultório foi bem-sucedida na remoção de pólipos endometriais $95,7 \%$ do tempo e que a histeroscopia na sala de cirurgia resultou em menos pólipos residuais, ou seja, em $98,1 \%$ não tiveram lesões residuais. Assim, considerando à sua eficácia (95,7\%), a conveniência, a flexibilidade no agendamento e a redução de custos associados à histeroscopia de consultório, muitas mulheres são receptivas a isso, mas deve-se levar em consideração a dor e o conforto das pacientes durante o procedimento. Onde a dor relatada pelas pacientes demonstrou ser razoável e tolerável, com uma pontuação média de 5 pontos na escala visual analógica, a escala clássica de dor variando de 0 a 10 (IMUDIA NA e NEW EP, 2019)

Quanto aos fatores de risco conhecidos para o câncer de endométrio seriam comuns para as mulheres com pólipos endometriais e espessamentos endometriais, e citam como exemplos idade, hipertensão, obesidade, diabetes e terapia hormonal (GHOUBARA, et al., 2018; BUOLENI-DIAS, et al., 2016) sendo considerados fatores preditivos para o desenvolvimento de pólipo endometrial (BUELONI-DIAS, et al., 2014). Outros estudos sugerem que a paridade, IMC, presença de mioma, uso de tamoxifeno, volume uterino, volume ovariano e estradiol sérico influenciam o espessamento endometrial (TE) em mulheres na pósmenopausa (HEBBAR S, et al., 2014).

Em casos de pólipos endometriais encontrados nas investigações realizadas através das ultrassonografias, investigando sangramento uterino anormal (AUB), são facilmente gerenciados através do procedimento da histeroscopia realizado em consultório. Dado o risco subjacente de malignidade nos pólipos endometriais, que pode ser tão baixo quanto $0,5 \%$ a $3 \%$ em todas as mulheres diagnosticadas com pólipos ou até $6 \%$ em mulheres pós-menopáusicas com sangramento, a remoção é fortemente recomendada com polipectomia histeroscópica para eventual diagnóstico histopatológico (IMUDIA NA e NEW EP, 2019).

\section{CONSIDERAÇÕES FINAIS}

O exame ultrassonográfico endovaginal apresenta diversas nuances no processo de avaliação das doenças endometriais, sendo um método de fácil acesso e baixo custo. Para avaliação das causas de sangramento na pós-menopausa, onde 0 aumento da espessura endometrial em mulheres pósmenopáusicas com ou sem AUB é principalmente devido a lesões benignas, como pólipos e miomas 
submucosos; a histeroscopia é um método considerado padrão ouro, seguro e confiável para avaliar e tratar essas lesões. Há ainda o uso da solução salina contraste sonohysterography (SCSH) para o diagnóstico de pólipos endometrial em mulheres com sangramento pós-menopausa, desde que o exame SCSH seja de ótima qualidade, então poderá ser considerado como um método para estratificar mulheres com sangramento pósmenopausa para posterior investigação diagnóstica e tratamento com histeroscopia. Como foi observado, há uma tendência atual de indicação de gestão individualizada para essas pacientes, devendo considerar as características de cada caso.

\section{REFERÊNCIAS}

1. ARAÚJO FM, et al. Artigo de revisão: pólipos endometriais. Rev Pat Tocantins. V. 3, n. 02, 2016

2. BILLINGSLEY CC, et al. The Use of Transvaginal Ultrasound in Type II Endometrial Cancer. International Journal of Gynecological Cancer, 2015; 25(5), 858-862. doi:10.1097/igc.0000000000000423

3. BUELONI-DIAS FN, et al. Metabolic syndrome as a predictor of endometrial polyps in postmenopausal women. The North American Menopause Society, 2016; 23(7), 759-764. doi: 10.1097/gme.0000000000000616.

4. BUELONI-DIAS FN, et al. Predictive factors for occurrence of endometrial polyps in postmenopausal women. Rev Bras Ginecol Obstet. 2014 Nov; 36(11):489-496.

5. CARVALHO GA. Câncer de Endométrio: quais os fatores associados com recidiva. Dissertação (Mestrado) -Universidade Federal do Rio Grande do Sul, Faculdade de Medicina, Programa de Pós-Graduação em Ciências da Saúde: Ginecologia e Obstetrícia, Porto Alegre, BR-RS, 2019.

6. CLARK TJ, STEVENSON H. Endometrial Polyps and Abnormal Uterine Bleeding (AUB-P): What is the relationship, how are they diagnosed and how are they treated? Best Practice \& Research Clinical Obstetrics \& Gynaecology, 2017, 40: 89-104.

7. FARIA F, FERNANDES ES. Perfil das pacientes investigadas por sangramento pós-menopausa no Hospital Júlia Kubitschek nos anos de 2010 a 2014. Revista Interdisciplinar de Estudos Experimentais-Animais e Humanos Interdisciplinary Journal of Experimental Studies, 2017; v. 9, n. 1.

8. FRITZ MA, et al. Endocrinologia e esterilidade ginecológica clínica. Wolters Kluwer Health / Lippincott Williams e Wilkins, 2011.

9. GONÇALVES KFS. Conduta em pólipos endometriais assintomáticos, revisão sistemática da literatura. TCC (Graduação - MEDICINA), Universidade Federal da Bahia, UNIVERSIDADE FEDERAL DA BAHIA, 2016.

10. GHOUBARA A, et al. Predictors of malignancy in endometrial polyps: study of 421 women with postmenopausal bleeding. Climacteric. 2017; Feb;21(1):82-87. doi: 10.1080/13697137.2017.1410783.

11. GHOUBARA A, et al. Thickened endometrium in asymptomatic postmenopausal women - determining an optimum threshold for prediction of atypical hyperplasia and cancer. Journal of Obstetrics Gynaecology. 2018 Nov; 38(8):11461149. doi: 10.1080/01443615.2018.1458081. Epub 2018 Jun 3.

12. HEBBAR S, et al. Factors influencing endometrial thickness in postmenopausal women. Ann Med Health Sci Res. 2014 Jul; 4 (4): 608-14. doi: 10.4103 / 2141-9248.139340.

13. IMUDIA NA, NEW EP. Endometrial polyps: In-office management. Contemporary OB/GYN, 2019; 64(09).

14. INCA. Estimativa 2018: incidência de câncer no Brasil / Instituto Nacional de Câncer José Alencar Gomes da Silva. Coordenação de Prevenção e Vigilância. [Internet]. Instituto Nacional de Câncer José Alencar Gomes da Silva. Rio de Janeiro; 2017. $128 \mathrm{p}$.

15. JIANG T, et al. Do endometrial lesions require removal? A retrospective study. BMC Women's Health, 2019; 19(1). doi:10.1186/s12905-019-0756-8

16. KORKMAZER E, et al. Hysteroscopic assessment of postmenopausal endometrial thickening. Prz Menopauzalny. 2014 Dec;13(6):330-3. doi: 10.5114/pm.2014.47985. Epub 2014 Dec 30.

17. MEENA J, et al. Story of a Giant Endometrial Polyp in Asymptomatic Postmenopausal Female. J Clin Diagn Res. 2017 Mar; 11 (3): QD06-QD07. doi: 10.7860 / JCDR / 2017 / 24801.9482. Epub 2017 mar 1.

18. MUNRO MG, et al. Os dois sistemas FIGO para sintomas normais e anormais de sangramento uterino e classificação de causas de sangramento uterino anormal nos anos reprodutivos: revisões de 2018. International Journal of Gynecology \& Obstetrics, 2018, 143.3: 393-408.

19. POGGIO GA, et al. Primeiro, o ultrassom: por que, como e quando? Rev. argent. radiol., Cidade Autônoma de Buenos Aires, 2017; v. 81, n. 3, p. 192-203, sept.

20. SADRO CT. Imaging the endometrium: A Pictorial Essay. Can Assoc Radiol J. 2016 Aug;67(3):254-62

21. SARVI F, et al. Histeroscopia: um método necessário para detectar patologias uterinas em mulheres pós-menopausa com sangramento uterino anormal ou aumento da espessura endometrial. Jornal turco de obstetrícia e ginecologia. 2016; 13 (4): 183-188. doi: 10.4274 / tjod.66674. 
22. SILVEIRA DF, et al. Avaliação ultrassonográfica no espessamento endometrial: revisão de literatura. Revista de Patologia do Tocantins; 2017, v. 4, n. 4, p. 60-64.

23. SINGH $P$, et al. Correlation of endometrial thickness with the histopathological pattem of endometrium in postmenopausal bleeding. J Obstet Gynaecology Índia. 2016 fev; 66 (1): 42-6. doi: 10.1007 / s13224-014-0627-z.

24. SPADOTO-DIAS D, et al. The value of hysteroscopic biopsy in the diagnosis of endometrial polyps. Womens Health (Lond). 2016 Jul;12(4):412-9. doi: 10.1177/1745505716653695.

25. WANDERLEY MD, et al. Accuracy of Transvaginal Ultrasonography, Hysteroscopy and Uterine Curettage in Evaluating Endometrial Pathologies. Rev Bras Ginecol Obstet. 2016 Oct; 38(10): 506-511.

26. VAN HANEGEM N, et al. Diagnostic workup for postmenopausal bleeding: a randomised controlled trial. [Miscellaneous] BJOG: An International Journal of Obstetrics \& Gynaecology. 124(6):987-988, May 2017.

27. VROOM AJ, et al. Diagnostic accuracy of saline contrast sonohysterography in detecting endometrial polyps in women with postmenopausal bleeding: systematic review and meta-analysis. Ultrasound Obstet Gynecol 2019; 54: 28-34 Published online 12 June 2019 in Wiley Online Library (wileyonlinelibrary.com). DOI: 10.1002/uog.20229. 